\title{
Adopting Nrf2 and NF-kB from cancer: Is there any role of the duo in diabetes?
}

Geeta Negi (M. S. Pharm), Ashutosh Kumar (Ph. D), Shyam S Sharma (Ph. D)*

Molecular Neuropharmacology Laboratory, Department of Pharmacology and Toxicology, National Institute of Pharmaceutical Education and Research (NIPER), Punjab, INDIA.

Running Title: Nrf2 and NF-kB in diabetes

\section{${ }^{\circledR}$ Correspondence:}

Dr. Shyam S. Sharma, Professor, Molecular Neuropharmacology Laboratory,

Department of Pharmacology and Toxicology,

National Institute of Pharmaceutical Education and Research (NIPER),

Sec-67, S.A.S.Nagar, Mohali, Punjab-160062, India.

Tel: +91-172-2214683-87; Fax: +91-172-2214692

E-mail: sssharma@niper.ac.in 


\begin{abstract}
Oxidative stress and inflammation are two well-recognized events occurring in normal cells during cancer chemotherapy. Several antioxidant and anti-inflammatory agents have therefore been tried as rational and effective strategies for chemoprotection. Elaborate studies on antioxidants and anti-inflammatory agents in various experimental models of cancer revealed that these agents act by modulating the expression and/or activity of transcription factors nuclear factor erythroid 2- related factor 2 (Nrf2) and nuclear factor-kappa B (NF$\kappa B)$. Nrf2 is considered main sentinel of antioxidant defence as it induces many antioxidant and phase 2 enzymes in response to stressful conditions. Likewise NF-kB is considered as denominator of chronic and acute inflammatory processes. In the recent years, it was found that a large pool of chemopreventive and chemoprotective agents that restrain NF-kB inflammatory cascade also activates Nrf2 signaling. Diabetes and complications thereof are also conditions of amplified oxidative stress and inflammation in various targets organs like retina, kidneys, nerves and vasculature. Here we propose that Nrf2 and NF-kB are involved in the etiopathogenesis of diabetes and thus form attractive therapeutic targets. Elucidation of potential involvement of Nrf2 and NF-kB pathways that are operative during multi-organ injury in diabetes may provide basis for development of novel therapies.
\end{abstract}

Key words: Cancer; Diabetes; Nrf2; NF-kB 


\section{Introduction}

Cancer chemoprevention is defined as a pharmacological approach to prevent or repress the process of carcinogenesis and chemoprotection is a term given to the therapeutic strategy to prevent the damage caused to the normal non-cancerous cell by cancer chemotherapeutic agents. Structurally and mechanistically different categories of natural and synthetic compounds have demonstrated cancer chemoprevention and protection by diminishing activation of carcinogens by metabolism and/or detoxifying the reactive electrophiles generated by carcinogens. Increased expression of various antioxidant and phase 2 detoxifying enzymes via Nrf2-ARE activation has been identified as one of the probable mechanism through which these agents exert their chemopreventive or chemoprotective actions [1]. Nrf2 is activated by stress caused by reactive species and leads to induction of a number of antioxidant enzymes (hemeoxygenase, $\mathrm{HO}-1$; glutamylcysteine synthetase, $\gamma^{-}$ GCS; glutathione peroxidise; glutathione reductase, and thioredoxin reductase 1) and phase II detoxifying enzymes (NAD(P)H:quinone oxidoreductase 1, NQO1; epoxide hydrolase; glutathione S-transferases; UDP-glucuronyltransferases) [2; 3; 4; 5]. Another factor that forms a major mechanism of chemoprevention and chemoprotection is inhibition of NF-kB cascade and downstream signalling. NF-kB is a pleiotropic factor from Rel family of transcription factors, which comprises of five distinct members: RelA (p65), RelB, c-Rel, p50/p105, and p52/p100. Once activated by a stimulus NF-kB binds to its target genes advancing towards transcription of diverse group of enzymes including inducible nitric oxide synthase (iNOS) and cyclooxygenase-2 (COX-2) and pro-inflammatory cytokines (Tumor necrosis factor-alpha TNF- $\alpha$ and Interleukines, IL) [6; 7; 8]. NF-kB has been found to be upregulated in normal cells during the process of carcinogenesis or during cancer chemotherapy. Thus, NF-кB inhibition surfaced as a lucrative target for cancer chemoprevention and protection. 
Although entirely different in etiogenesis and progression, diabetes is related to cancer in two prospects- having oxidative stress and inflammation associated with its pathophysiology. Many of the critical pathways of pathophysiology of diabetic complications namely polyol pathway, advanced glycated end products (AGE) formation, protein kinase C (PKC) activation, hexosamine pathways, PARP overactivation, mitogen activated protein kinase activation, etc. have oxidative stress as common feature. As mentioned above Nrf2 is a key transcription factor involved in combating oxidative stress. Apart from its protective role in cancer, it has also been postulated to be a significant defending factor in diabetes and its complications [9; 10]. In some recent findings it was established that Nrf2 is crucial in the modulation of insulin resistance and diabetes [11; 12; 13].

Diabetes has been known for decades to trigger chronic inflammation as ample evidence are there in literature to prove that inflammation is an important pathogenic mechanism in the development of diabetic complications [14]. Elevated CRP (C-reactive protein, an inflammatory biomarker) levels significantly predicted the risk of developing type 2 diabetes, and this risk was independent of other factors [15]. NF-кB signaling cascade constitutes an attractive target for anti-inflammatory therapeutic interventions. There are upcoming evidences to support the role of NF-kB inflammatory cascade in the diabetogenesis and in the aetiology of various diabetic complications [16; 17]. The critical role of NF-kB activation in pathophysiology of diabetic complications and the merit of inhibiting NF- $\mathrm{KB}$ in correcting diabetic abnormalities indicate that targeting NF-kB is a desired therapeutic strategy for the treatment of diabetic complications.

In the present review, we put forward scheme that since oxidative stress and inflammation play a pivotal role in the development and progression of diabetic complications, Nrf2 and NF-kB are major players here too (Fig 1). Furthermore, we suggest that Nrf2 
activation/overexpression and NF-kB inhibition may provide an attractive drug target for development of therapeutic interventions for diabetes related complications.

\section{Nrf2: Protection against oxidative stress in cancer and diabetes}

A fairly large number of antioxidants and phase 2 detoxifying enzymes that form a line of resistance against toxic chemicals like chemotherapeutic agents, are transcriptionaly regulated through a cis-acting DNA regulatory element termed antioxidant response element. Numerous studies have demonstrated that many of the chemopreventive agents act through Nrf2 system to augment ARE-driven gene expression [18; 19].

Nrf-2 is a member of cap 'n' collar basic region leucine zipper (CNC-bZIP) family of transcription factors [20]. Of many transcription factors activating ARE, Nrf2 has been recognized as the key signaling system leading to ARE mediated transcription. As other transcription factors, in physiological conditions Nrf2 is under negative regulation in the cytoplasm. Kelch-like ECH-associated protein (Keap1) has been identified as the negative regulator of Nrf2. Exposure of cells to reactive electrophiles results in the covalent modification of cysteine thiol groups on Keap1 resulting in a conformational change in Keap1 structure. This event result in the release of free Nrf2 which consequently translocates to the nucleus to interact with ARE to turn on the transcription of genes for antioxidant and detoxification enzymes [21; 22; 23].

Extensive research in the field of cancer biology and pathomechanisms has led to many innovative strategies for cancer treatment. Rapid inactivation and elimination of carcinogens or enhanced cellular antioxidant defence against reactive species generated from these agents have been identified as rational approaches for chemoprotection. Many phytochemicals have been long known for their phase 2 detoxifying enzyme inducing activity and interestingly many of these have been identified to enhance ARE driven gene transcription through 
transcription factor Nrf2. Oltipraz, a synthetic analog of dithiolethiones isolated from cruciferous vegetables, has emerged as a prototypical cancer chemopreventive agent [24]. It has been found to be a potential agent in preventing carcinogen-induced tumorigenesis in various experimental models[25]. Oltipraz has completed a few clinical trials on cancer chemoprevention against the carcinogenic metabolites of aflatoxin [26] and is currently being tried for safety and efficacy with other types of carcinogens. Besides oltipraz a number of natural agents and their synthetic derivatives which are currently being tried in cancer have been elucidated to activate Nrf2 signaling. Examples include curcumin (a traditional spice), epigallocatechin-3-gallate (polyphenols extracted from green tea), sulforaphane (isothiocyanate constituent of crucifers), resveratrol (polyphenol in grapes and wine), allyl sulfides (organosulphur content of garlic oil), xanthohumol (sesquiterpene derived from hops), cafestol (coffee derived diterpene), lycopene (an antioxidant in tomato), carnosol (orthophenolic diterpene in rosemary), zerumbone (sesquiterpene in ginger), chalcones ( $\alpha, \beta$ unsaturated flavanoids), vanilloids (pungent ingredients of capsicum and ginger) etc [27]. Apart from studies with the above agents in cancer, Nrf2 knockout animals provide a direct evidence of Nrf2-mediated protection against cancer. Nrf2 knockout mice display an increased vulnerability to chemical carcinogenesis and as well as reduced induction of phase 2 enzymes in response to Nrf2 activating chemoprotective agents [28; 29]. Thus, discovery of Nrf2 as a curator of antioxidant and detoxification enzymes levels has created a new podium for the design and development of a novel category of therapeutic chemopreventive intervention.

Similar to cancer, in diabetes also a general consent on its pathophysiology has evolved with increased oxidative and inflammatory stress forming the core. The onus now is to delineate the precise factors and pathways responsible for the generation of oxidative damage, as well as the inflammation in diabetes. Nrf2 is considered as the axis of defence against oxidative stress, 
there is a definite correlation between pathogenesis of diabetic complications and Nrf2 signaling. Many of the cancer chemopreventive agents have found their way in diabetes research on the grounds that they protect the cell against oxidative stress by increasing Nrf2ARE interaction. Pancreatic $\beta$ cells are particularly susceptible to cytokines induced production of ROS and oxidative damage resulting in insulin deficiency and type 1 diabetes.

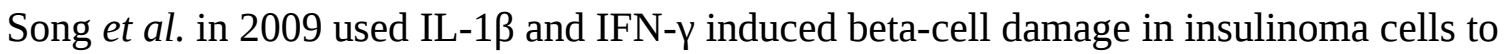
mimic this condition. It was observed that sulforaphane an Nrf2 activator prevented the cytokine toxicity and inhibited cytokine-induced $\mathrm{H}_{2} \mathrm{O}_{2}$ production [30]. Xue et al. in 2008 reported the reversal of various dysfunctions in human microvascular HMEC-1 endothelial cells under high glucose conditions by sulforaphane which implies that Nrf2 activation could be adopted as a strategy to reverse the development of macro- and microvascular complication of diabetes [12]. Ebselen a glutathione peroxidase mimetic and a cancer chemoprotectant prevented vascular dysfunction and apoptosis in animal model of type 2 diabetes by increasing HO-1 expression (an antioxidant enzyme regulated by Nrf2) [31]. A series of experiments with Nrf2 knock out animals also favour the involvement of Nrf2 in diabetic condition. In a study by Aleksunes et al. in 2010, it was observed that Nrf2 knock out diabetic animals had higher blood glucose levels than wild-type diabetic animals and oltipraz was found effective in lowering blood glucose in wild-type diabetic but not in Nrf2 deleted diabetic animals [32]. Similarly knock out models provide a clue on various other complications as well like diabetic cardiomyopathy, nephropathy and neuropathy [33]. Since background literature support the protective role of Nrf2 in animal models of diabetes and its complications, exploiting this pathway by various activators may pave a way to the management of diabetes related complications.

NF- KB: Initiator and propagator of inflammation in cancer and diabetes 
NF-kB is one of the best-characterized transcription factors expressed ubiquitously. Its deregulation has been associated with many disease conditions like cancer, diabetes, AIDS, atherosclerosis, asthma, arthritis, inflammatory bowel disease, muscular dystrophy, stroke and viral infections. It regulates the expression of many proteins in normal and cancer cells and provides a connecting bridge between inflammation and cancer. After nearly two decades of intense research, it is now clear that NF-kB represents a realistic molecular target for the development of effective cancer therapies [34].

In normal condition, NF-kB is sequestered within the cytosol by an inhibitory protein (i.e., IкB; inhibitor of NF-кB) that masks the nuclear localization signal present within the NF-кB protein and keeps it in inactive form. TNF- $\alpha$ and IL-1, or bacterial products such as lipopolysaccharide (LPS), can activate the specific IкB kinase (IKK) complex that in turn phosphorylates IкB. The phosphorylated IкB is tagged by ligases and ubiquitinated which is a signal for its degradation by the proteasome [35; 36]. The degradation of IкB frees p65/p50 dimer which can translocate into the nucleus where it can act as a transcription factor and can turn on the transcription of various genes for proinflammatory mediators and inducible enzymes [37].

NF-kB overactivation acts as a savior for many of tumor types by executing its antiapoptotic effect in the tumor cells. Deregulated NF-kB activation is a hallmark of several lymphoid malignancies and other cancer types [38; 39; 40]. This dysregulation causes an increase in the levels of proteins responsible for angiogenesis and proliferation, protection from radiation and chemotherapy induced apoptosis and helping tumor cells to develop resistance to therapies used. There are ample preclinical and clinical reports which points towards the notion that inhibiting NF-kB can aid to the existing therapies or the methods that are being used to provide long term remission. NF-кB inhibitors like BAY 11-7082, MG-132, theoflavins, PS-341 and curcumin have showed beneficial effect against various in vitro 
models of cancers. MG-132 an inhibitor of NF-kB proteasomal degradation inhibited the proliferation and angiogenesis in breast cancer cells in in vitro experimentation and hence can be a good futuristic option for treating breast cancers [41]. Besides having direct effect on cancer cells, NF-kB also forms an excellent target for cancer chemoprevention. Similar to their influence on Nrf2, large number of chemoprotective agents can regulate the altered level

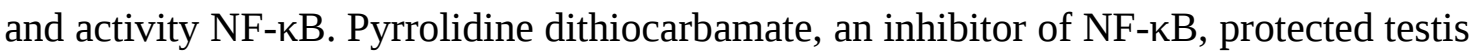
against the damage caused by cisplatin [42]. Agents which are known Nrf2 activators have also been demonstrated to possess NF-kB inhibitory activity thus affording dual protection from chemotherapy-induced damage to various target organs. Examples include resveratrol, curcumin, epigallocatechin gallate and silymarin [43].

There are upcoming evidences to support the role of NF-kB inflammatory cascade in the diabetogenesis and in the etiology of various diabetic complications [16; 17]. Inhibition of IкB kinase 2 using AS602868, alleviated nitric oxide-dependent diabetic erectile dysfunction which suggests that NF-kB activation is an important contributor to development of diabetic cavernosum nitrergic neuropathy and vasculopathy [44]. NF-кB has been identified as a key feature in the pathogenesis of diabetic neuropathy [45]. Activation of NF-kB in ex vivo isolated peripheral blood mononuclear cells of patients with diabetes mellitus was correlated with severity of diabetic nephropathy. In a clinical study, NF-kB signaling cascades and downstream effectors were found overtly active in kidneys of diabetic subject [46]. NF-kB also contributes toward interstitial inflammation and tubular interstitial fibrosis in the rat obstructive nephropathy which points towards possible role of NF-kB inflammatory cascade in nephropathy [47]. NF-kB has also been known to contribute towards pathophysiology of diabetic retinopathy where it serves to regulate the expression of angiogenic factors [48]. However, before forming any conclusion on the role of NF-kB in diabetogenesis and diabetic 
complication, further experimesntal and clinical investigations about the precise role of NFкB in pancreatic $\beta$ cells and all cell types affected by hyperglycemia are required.

\section{The other side of the coin}

With the growing interest and research on Nrf2 and NF-kB, interestingly latest reports have exposed the other side of the coin. Nrf2 is not only involved in conferring protection to normal cells from oxidative stress and putting a check on their cancer transformation, but also imparts resistance to the cancerous cells to chemo- or radiotherapy. Few reports have also speculated that Nrf2 plays a role of promoter in carcinogenesis [50; 51]. Some cancer cells or tumors have been found to under express Keap1 (negative regulator of Nrf2) or have mutation or alterations in Keap1 [52]. In atherosclerosis also, it was found that Nrf2 knockout leads to atherosclerotic progression [53]. In diabetes such direct evidences are not yet available but considering the reports from other pathophysiological conditions, a concrete conclusion cannot be derived that Nrf2 activation will produce only the desired beneficial effects and will not aggravate the pathological state. Likewise, NF-kB is involved in the regulation of a broad range of biological processes in immune system such as inflammatory response, apoptosis and in central nervous system such as synaptic plasticity, neurogenesis, and differentiation [54; 55]. NF-кB represents an extremely attractive target for designing therapeutic interventions, but it remains a challenging task for drug discovery people to design inhibitors that block NF-kB in a given setting, while sparing the physiological function in other context so that the balanced NF-kB activation/activity is maintained between immune homeostasis and regulation of appropriate anti-inflammatory response. It appears that the black and white concept of NF-kB and Nrf2 just represents the one eyed view of both the factors. Comprehensive knowledge on triggering stimuli, physiological role, signaling network and downstream targets of NF-kB and Nrf2 can lead to a better perception on their double edged sword character. 


\section{Acknowledgement}

The authors would like to acknowledge the financial support provided by Department of Pharmaceuticals, Ministry of Chemicals and Fertilizers, Government of India to Dr. S.S. Sharma. Ms Geeta Negi is a recipient of CSIR-NET research fellowship.

\section{Conflicts of Interest}

The authors declare no conflicts of interest.

\section{Figure Legends}

Fig 1: Nrf2 and NF-kB pathways and proposed role in diabetic complications. Oxidative stress and inflammation are the two major pathways associated with most of the complications of diabetes, retinopathy, nephropathy, neuropathy, vasculopathy etc. As a well known and well proven fact, long term hyperglycemia results in the generation of ROS causing oxidative stress. Since Nrf2 is the key transcription factor involved in maintaining antioxidant enzymes levels (HO-1, NQO-1 and SOD), it is proposed that in diabetes decreased expression of Nrf-2 compromises the redox status of cell which culminates in oxidative stress. Hyperglycemia-led low grade inflammation forms another basis for diabetic complications. NF-kB p65 subunit activated by factors generated by chronic high glucose environment generates inflammatory mediators (TNF- $\alpha$ and interleukins) and enzymes linked to inflammation (iNOS and COX-2) which direct cells towards inflammatory injuries.

\section{References}

[1] Y. Zhang, and G.B. Gordon, A strategy for cancer prevention: stimulation of the Nrf2ARE signaling pathway. Mol. Cancer Ther. 3 (2004) 885-893.

[2] M. Kobayashi, and M. Yamamoto, Molecular mechanisms activating the Nrf2-Keap1 pathway of antioxidant gene regulation. Antioxid. Redox Signal. 7 (2005) 385-94. 
[3] W. Li, and A.N. Kong, Molecular mechanisms of Nrf2-mediated antioxidant response. Mol. Carcinog. 48 (2009) 91-104.

[4] A.T. Dinkova-Kostova, and P. Talalay, NAD(P)H:quinone acceptor oxidoreductase 1 (NQO1), a multifunctional antioxidant enzyme and exceptionally versatile cytoprotector. Arch. Biochem. Biophys. 501 (2010) 116-123.

[5] J.W. Kaspar, S.K. Niture, and A.K. Jaiswal, Nrf2:INrf2 (Keap1) signaling in oxidative stress. Free Radic. Biol. Med. 47 (2009) 1304-1309.

[6] M. Karin, Y. Yamamoto, and Q.M. Wang, The IKK NF-kappa B system: a treasure trove for drug development. Nat. Rev. Drug Discov. 3 (2004) 17-26.

[7] S. Rosales-Corral, R.J. Reiter, D.X. Tan, G.G. Ortiz, and G. Lopez-Armas, Functional aspects of redox control during neuroinflammation. Antioxid. Redox Signal. 13 (2010) 193-247.

[8] A.S. Shifera, Proteins that bind to IKKgamma (NEMO) and down-regulate the activation of NF-kappaB. Biochem. Biophys. Res. Commun. 396 (2010) 585-589.

[9] G. Negi, A. Kumar, R.P. Joshi, and S.S. Sharma, Oxidative stress and Nrf2 in the pathophysiology of diabetic neuropathy: Old perspective with a new angle. Biochem. Biophys. Res. Commun. 408 (2011) 1-5.

[10] G. Negi, A. Kumar, and S.S. Sharma, Melatonin modulates neuroinflammation and oxidative stress in experimental diabetic neuropathy: effects on NF-kappaB and Nrf2 cascades. J. Pineal Res. 50 (2011) 124-131.

[11] B.B. Aggarwal, Targeting inflammation-induced obesity and metabolic diseases by curcumin and other nutraceuticals. Annu. Rev. Nutr. 30 (2010) 173-199.

[12] M. Xue, Q. Qian, A. Adaikalakoteswari, N. Rabbani, R. Babaei-Jadidi, and P.J. Thornalley, Activation of NF-E2-related factor-2 reverses biochemical dysfunction of 
endothelial cells induced by hyperglycemia linked to vascular disease. Diabetes 57 (2008) 2809-2817.

[13] K. Yoh, A. Hirayama, K. Ishizaki, A. Yamada, M. Takeuchi, S. Yamagishi, N. Morito, T. Nakano, M. Ojima, H. Shimohata, K. Itoh, S. Takahashi, and M. Yamamoto, Hyperglycemia induces oxidative and nitrosative stress and increases renal functional impairment in Nrf2-deficient mice. Genes Cells 13 (2008) 1159-1170.

[14] M.Y. Donath, and S.E. Shoelson, Type 2 diabetes as an inflammatory disease. Nat. Rev. Immunol. 11 98-107.

[15] D.J. Freeman, J. Norrie, N. Sattar, R.D. Neely, S.M. Cobbe, I. Ford, C. Isles, A.R. Lorimer, P.W. Macfarlane, J.H. McKillop, C.J. Packard, J. Shepherd, and A. Gaw, Pravastatin and the development of diabetes mellitus: evidence for a protective treatment effect in the West of Scotland Coronary Prevention Study. Circulation 103 (2001) 357-362.

[16] N.E. Cameron, and M.A. Cotter, Pro-inflammatory mechanisms in diabetic neuropathy: focus on the nuclear factor kappa B pathway. Curr. Drug Targets 9 (2008) 60-67.

[17] G. Michalowska-Wender, G. Adamcewicz, and M. Wender, Impact of cytokines on the pathomechanism of diabetic and alcoholic neuropathies. Folia Neuropathol. 45 (2007) 78-81.

[18] W. Wang, and A.K. Jaiswal, Nuclear factor Nrf2 and antioxidant response element regulate NRH:quinone oxidoreductase 2 (NQO2) gene expression and antioxidant induction. Free Radic. Biol. Med. 40 (2006) 1119-1130.

[19] J. Alam, D. Stewart, C. Touchard, S. Boinapally, A.M.K. Choi, and J.L. Cook, Nrf2, a Cap n Collar Transcription Factor, Regulates Induction of the Heme Oxygenase-1 Gene. J. Biol. Chem. 274 (1999) 26071-26078. 
[20] M. McMahon, K. Itoh, M. Yamamoto, S.A. Chanas, C.J. Henderson, L.I. McLellan, C.R. Wolf, C. Cavin, and J.D. Hayes, The Cap and Collar Basic Leucine Zipper Transcription Factor Nrf2 (NF-E2 p45-related Factor 2) Controls Both Constitutive and Inducible Expression of Intestinal Detoxification and Glutathione Biosynthetic Enzymes. Cancer Research 61 (2001) 3299-3307.

[21] A.K. Jaiswal, Nrf2 signaling in coordinated activation of antioxidant gene expression. Free Radic. Biol. Med. 36 (2004) 1199-1207.

[22] K.A. Jung, and M.K. Kwak, The Nrf2 system as a potential target for the development of indirect antioxidants. Molecules 15 (2010) 7266-7291.

[23] N. Wakabayashi, S.L. Slocum, J.J. Skoko, S. Shin, and T.W. Kensler, When Nrf2 Talks, Who's Listening? Antioxid. Redox Signal. 13 (2010) 1649-1663.

[24] T.W. Kensler, J.D. Groopman, T.R. Sutter, T.J. Curphey, and B.D. Roebuck, Development of cancer chemopreventive agents: oltipraz as a paradigm. Chem. Res. Toxicol. 12 (1999) 113-126.

[25] Y.L. Chua, D. Zhang, U. Boelsterli, P.K. Moore, M. Whiteman, and J.S. Armstrong, Oltipraz-induced phase 2 enzyme response conserved in cells lacking mitochondrial DNA. Biochem. Biophys. Res. Commun. 337 (2005) 375-381.

[26] J.S. Wang, X. Shen, X. He, Y.R. Zhu, B.C. Zhang, J.B. Wang, G.S. Qian, S.Y. Kuang, A. Zarba, P.A. Egner, L.P. Jacobson, A. Munoz, K.J. Helzlsouer, J.D. Groopman, and T.W. Kensler, Protective alterations in phase 1 and 2 metabolism of aflatoxin B1 by oltipraz in residents of Qidong, People's Republic of China. J. Natl. Cancer Inst. 91 (1999) 347-354.

[27] C.R. Zhao, Z.H. Gao, and X.J. Qu, Nrf2-ARE signaling pathway and natural products for cancer chemoprevention. Cancer Epidemiol. 34 (2010) 523-533. 
[28] L. Becks, M. Prince, H. Burson, C. Christophe, M. Broadway, K. Itoh, M. Yamamoto, M. Mathis, E. Orchard, R. Shi, J. McLarty, K. Pruitt, S. Zhang, and H.E. KleinerHancock, Aggressive mammary carcinoma progression in Nrf2 knockout mice treated with 7,12-dimethylbenz[a]anthracene. BMC Cancer 10 (2010) 540.

[29] T.O. Khor, M.T. Huang, A. Prawan, Y. Liu, X. Hao, S. Yu, W.K. Cheung, J.Y. Chan, B.S. Reddy, C.S. Yang, and A.N. Kong, Increased susceptibility of Nrf2 knockout mice to colitis-associated colorectal cancer. Cancer Prev. Res. (Phila) 1 (2008) 187191.

[30] M.Y. Song, E.K. Kim, W.S. Moon, J.W. Park, H.J. Kim, H.S. So, R. Park, K.B. Kwon, and B.H. Park, Sulforaphane protects against cytokine- and streptozotocin-induced beta-cell damage by suppressing the NF-kappaB pathway. Toxicol. Appl. Pharmacol. 235 (2009) 57-67.

[31] A.L. Kruger, S.J. Peterson, M.L. Schwartzman, H. Fusco, J.A. McClung, M. Weiss, S. Shenouda, A.I. Goodman, M.S. Goligorsky, A. Kappas, and N.G. Abraham, Upregulation of heme oxygenase provides vascular protection in an animal model of diabetes through its antioxidant and antiapoptotic effects. J. Pharmacol. Exp. Ther. 319 (2006) 1144-1152.

[32] L.M. Aleksunes, S.A. Reisman, R.L. Yeager, M.J. Goedken, and C.D. Klaassen, Nuclear factor erythroid 2-related factor 2 deletion impairs glucose tolerance and exacerbates hyperglycemia in type 1 diabetic mice. J. Pharmacol. Exp. Ther. 333 (2010) 140-151.

[33] R. Pazdro, and J.R. Burgess, The role of vitamin E and oxidative stress in diabetes complications. Mech. Ageing Dev. 131 (2010) 276-286.

[34] P.J. Jost, and J. Ruland, Aberrant NF-kappaB signaling in lymphoma: mechanisms, consequences, and therapeutic implications. Blood 109 (2007) 2700-2707. 
[35] S. Ghosh, M.J. May, and E.B. Kopp, NF-kappa B and Rel proteins: evolutionarily conserved mediators of immune responses. Annu. Rev. Immunol. 16 (1998) 225-260.

[36] M. Karin, and Y. Ben-Neriah, Phosphorylation meets ubiquitination: the control of NF[kappa]B activity. Annu. Rev. Immunol. 18 (2000) 621-663.

[37] F. D'Acquisto, M.J. May, and S. Ghosh, Inhibition of nuclear factor kappa B (NF-B): an emerging theme in anti-inflammatory therapies. Mol. Interv. 2 (2002) 22-35.

[38] K.A. Higgins, J.R. Perez, T.A. Coleman, K. Dorshkind, W.A. McComas, U.M. Sarmiento, C.A. Rosen, and R. Narayanan, Antisense inhibition of the p65 subunit of NF-kappa B blocks tumorigenicity and causes tumor regression. Proc. Natl. Acad. Sci. USA 90 (1993) 9901-9905.

[39] T. Nishina, N. Yamaguchi, J. Gohda, K. Semba, and J.-i. Inoue, NIK is involved in constitutive activation of the alternative NF-kB pathway and proliferation of pancreatic cancer cells. Biochem. Biophys. Res. Commun. 388 (2009) 96-101.

[40] E. Pikarsky, R.M. Porat, I. Stein, R. Abramovitch, S. Amit, S. Kasem, E. GutkovichPyest, S. Urieli-Shoval, E. Galun, and Y. Ben-Neriah, NF-kappaB functions as a tumour promoter in inflammation-associated cancer. Nature 431 (2004) 461-466.

[41] A. Shibata, T. Nagaya, T. Imai, H. Funahashi, A. Nakao, and H. Seo, Inhibition of NFkappaB activity decreases the VEGF mRNA expression in MDA-MB-231 breast cancer cells. Breast Cancer Res. Treat. 73 (2002) 237-243.

[42] Y.O. Ilbey, E. Ozbek, A. Simsek, M. Cekmen, A. Otunctemur, and A. Somay, Chemoprotective effect of a nuclear factor-kappaB inhibitor, pyrrolidine dithiocarbamate, against cisplatin-induced testicular damage in rats. J. Androl. 30 (2009) 505-514.

[43] R. Ralhan, M.K. Pandey, and B.B. Aggarwal, Nuclear factor-kappa B links carcinogenic and chemopreventive agents. Front. Biosci. (Schol. Ed.) 1 (2009) 45-60. 
[44] M.R. Nangle, M.A. Cotter, and N.E. Cameron, IkappaB kinase 2 inhibition corrects defective nitrergic erectile mechanisms in diabetic mouse corpus cavernosum. Urology 68 (2006) 214-218.

[45] A. Kumar, G. Negi, and S.S. Sharma, JSH-23 targets nuclear factor kappa B (NFkappaB) and reverses various deficits in experimental diabetic neuropathy: effect on neuroinflammation and antioxidant defence. Diabetes Obes. Metab. 13 (2011) 750758.

[46] H. Schmid, A. Boucherot, Y. Yasuda, A. Henger, B. Brunner, F. Eichinger, A. Nitsche, E. Kiss, M. Bleich, H.J. Grone, P.J. Nelson, D. Schlondorff, C.D. Cohen, and M. Kretzler, Modular activation of nuclear factor-kappaB transcriptional programs in human diabetic nephropathy. Diabetes 55 (2006) 2993-3003.

[47] K. Tashiro, S. Tamada, N. Kuwabara, T. Komiya, K. Takekida, T. Asai, H. Iwao, K. Sugimura, Y. Matsumura, M. Takaoka, T. Nakatani, and K. Miura, Attenuation of renal fibrosis by proteasome inhibition in rat obstructive nephropathy: possible role of nuclear factor kappaB. Int. J. Mol. Med. 12 (2003) 587-592.

[48] G. Romeo, W.H. Liu, V. Asnaghi, T.S. Kern, and M. Lorenzi, Activation of nuclear factor-kappaB induced by diabetes and high glucose regulates a proapoptotic program in retinal pericytes. Diabetes 51 (2002) 2241-2248.

[49] O. Lorenzo, B. Picatoste, S. Ares-Carrasco, E. Ramirez, E.R. Jesus, and J. Tunon, Potential role of Nuclear Factor kB in Diabetic Cardiomyopathy. Exp. Diabetes Res. In press (2011).

[50] T.W. Kensler, and N. Wakabayashi, Nrf2: friend or foe for chemoprevention? Carcinogenesis 31 (2010) 90-99.

[51] A. Lau, N.F. Villeneuve, Z. Sun, P.K. Wong, and D.D. Zhang, Dual roles of Nrf2 in cancer. Pharmacol. Res. 58 (2008) 262-270. 
[52] A. Singh, V. Misra, R.K. Thimmulappa, H. Lee, S. Ames, M.O. Hoque, J.G. Herman, S.B. Baylin, D. Sidransky, E. Gabrielson, M.V. Brock, and S. Biswal, Dysfunctional KEAP1-NRF2 interaction in non-small-cell lung cancer. PLoS Med. 3 (2006) e420.

[53] T.E. Sussan, J. Jun, R. Thimmulappa, D. Bedja, M. Antero, K.L. Gabrielson, V.Y. Polotsky, and S. Biswal, Disruption of Nrf2, a key inducer of antioxidant defenses, attenuates ApoE-mediated atherosclerosis in mice. PLoS One 3 (2008) e3791.

[54] E.D. Foehr, X. Lin, A. O'Mahony, R. Geleziunas, R.A. Bradshaw, and W.C. Greene, NF-kappa B signaling promotes both cell survival and neurite process formation in nerve growth factor-stimulated PC12 cells. J. Neurosci. 20 (2000) 7556-7563.

[55] A. Kumar, Y. Takada, A.M. Boriek, and B.B. Aggarwal, Nuclear factor-kappaB: its role in health and disease. J. Mol. Med. 82 (2004) 434-448. 
Fig 1

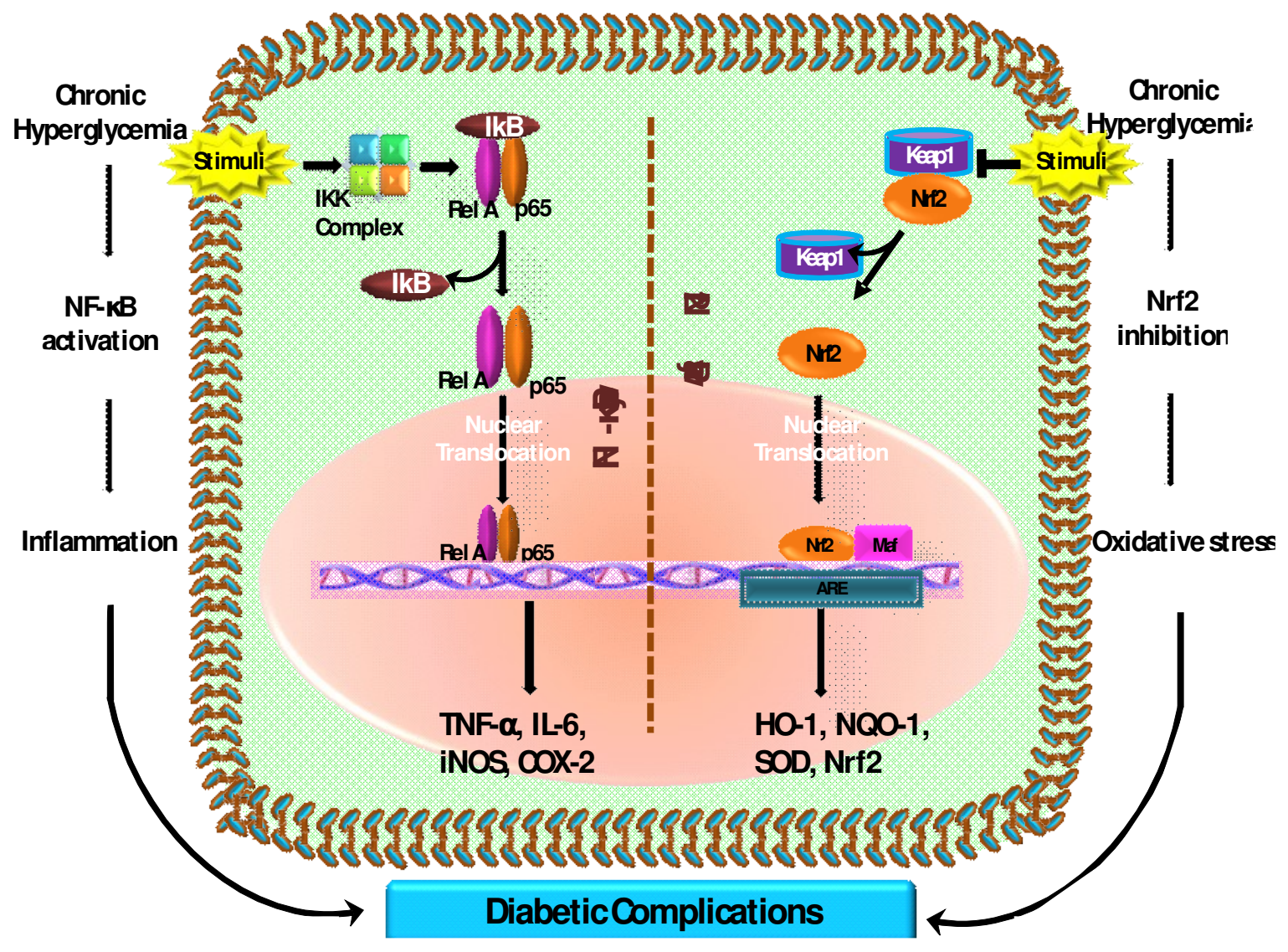

